\title{
Challenges of Investigating Fluid-Elastic Lock-In of a Shallow Cavity and a Cantilevered Beam at Low Mach Numbers
}

\author{
K Lai-Fook Cody, S Hambirc, M Pollack
}

This report was prepared as an account of work sponsored by the United States Government. Neither the United States, nor the United States Department of Energy, nor any of their employees, nor any of their contractors, subcontractors, or their employees, makes any warranty, express or implied, or assumes any legal liability or responsibility for the accuracy, completeness or usefulness of any information, apparatus, product or process disclosed, or represents that its use would not infringe privately owned rights. 


\section{CHALLENGES OF INVESTIGATING FLUID-ELASTIC LOCK-IN OF A SHALLOW CAVITY AND A CANTILEVERED BEAM AT LOW MACH NUMBERS}

\author{
Kristin Lai-Fook Cody \\ Lockheed-Martin, Inc. \\ PO Box 1072 \\ Schenectady, NY 12301
}

\author{
Stephen A. Hambric \\ ARL/Penn State \\ PO Box 30 \\ State College, PA 16804
}

\author{
Martin L. Pollack \\ Lockheed-Martin, Inc. \\ PO Box 1072 \\ Schenectady, NY 12301
}

\begin{abstract}
At low flow Mach numbers, fluid-elastic lock-in may occur when a shear layer instability interacts with an adjoining or nearby structure and the resulting vibration of the structure reinforces the shear layer instability. Despite the significant amount of study of lock-in with acoustic resonators, fluidelastic lock-in of a shear layer fluctuation over a cavity and a structural resonator is not well understood and has not been thoroughly studied. Design of an experimental system is described and preliminary diagnostics are addressed as a basis for a platform for developing a fundamental understanding of the feedback mechanism, analytical models for predicting and describing fluid-elastic lock-in conditions, and the roles of the fluid and structural dynamics in the process. Features of the system investigated here include design for characterization of modal excitation of a beam-like structure from the shear layer fluctuation, isolation of the predominant instability source to the shear layer fluctuation over the cavity, variation of the cavity size to identify critical parameters that govern fluidelastic lock-in, and alteration of the inflow boundary layer momentum thickness. So far, lock-in between the cavity and the distributed elastic resonator has not been achieved. Further investigations to determine the role of the source and resonator attributes are underway.
\end{abstract}

\section{INTRODUCTION}

Complex behavior can result from fluid flow interacting with a structure. A wide variety of research in the fields of aeroelasticity and hydrodynamics has generated numerous results useful in predicting and describing fluid-structure interaction. In flow-induced vibrations, hydrodynamic forces interact with the elastic properties of a structure, resulting in fluid-elastic interaction. Flow interacting with a structure produces vortices which shed at a prescribed frequency. If the shedding frequency coincides with the resonance frequency of an adjoining elastic structure or acoustic fluid volume, the resulting oscillation can reinforce the vorticity, creating a feedback mechanism responsible for producing high energy at resonance. This phenomenon is called lock-in and at low Mach numbers can result in a strong narrowband sound source. Lock-in is often undesirable and can promote rapid fatigue failure.

Lock-in can occur between many different kinds of fluid instabilities and acoustic or structural resonances, and thus is prevalent in many different situations. One application not well understood is referred to as fluid-elastic lock-in which is the locking in of an unstable shear layer created from flow over a cavity with the structural resonance of a body containing the cavity or contained within the cavity. Existing research describes cases of lock-in at low Mach number and distinguishes it from other aeroelastic and hydrodynamic instabilities, but does not fully capture the physics of the feedback mechanism for the cavity structural lock-in situation.

Other types of cavity lock-in have been studied extensively. There are three main categories of fluid interaction mechanisms for cavity oscillations (Rockwell and Naudascher, 1978). Each interaction differs in the feedback mechanism to the fluid source. Fluid-dynamic interaction is the feedback of the shear layer flow from either side of the cavity alone. Fluidresonant interaction occurs when the shear layer cavity oscillation is coupled to acoustic resonant wave effects in the cavity. Fluid-resonant interaction is also prevalent for acoustic resonances external to the cavity such as pipes containing the cavity. Both types of fluid-resonant lock-in are shown in Figure 1. Fluid-elastic interaction occurs when a wall of the cavity is able to displace and provide a feedback path to the shear layer oscillation. Very limited information exists for this type of fluid-elastic cavity lock-in (Dunham, 1962; Harrington and Dunham, 1960). No information has been found for lockin with an elastic resonator external to the cavity which acts more as a distributed mass resonator compared to the localized cavity source. Both types of fluid-elastic lock-in are shown in Figure 1. Most systems are actually a combination of one or all categories, but are better described in one concentrated area. 
Much literature exists in the study of fluid-dynamic and fluidresonant cases, but fluid-elastic cases are more limited in possibilities and are more complex due to the coupling of the dynamic characteristics from the structure and the flow. The elastic properties of the structures in the cavity oscillation situations do have an effect on the fluid-dynamic and fluidresonant categories. By studying the fluid-elastic category in a concentrated manner, results can be applied to models developed for the fluid-dynamic and fluid-resonant cases.

Although elastic interaction with a cavity source has not been widely studied, observations from other types of impingement interactions with elastic interactions may be useful. One impingement case of interest is a jet followed by a downstream edge, commonly referred to as a jet-edge or edgetone generator (Figure 2). In both jet-edge and cavity oscillations, impingement provides a feedback mechanism, in which downstream impingement is felt instantaneously at the upstream edge. At low Mach numbers, the impingement distance relative to acoustic wavelength is much less than one, so the downstream source is in a non-propagating region and the upstream influence and feedback occur in a hydrodynamic manner. The phase-lock condition describes frequencies of this feedback which will be used in this study as a design parameter for the experiment.

The principle of elastic feedback has been more extensively studied with jets rather than cavities. When a jet impinges on an elastically mounted wedge, the periodicity of the vortex generation provides the frequency interaction for lock-in. The primary vortices distort the wedge position and secondary vortices are created which then feedback on the primary source. The feedback is then strengthened by the wedge oscillation. A similar situation occurs for cavities. The incompressible feedback due to impingement and upstream influence is strengthened when the frequency associated with this is locked into a nearby acoustic or structural resonance frequency, producing a self-sustaining interaction.

Elastic feedback with other types of fluid sources has been extensively studied, such as elastically mounted bluff body instability and blade trailing edge instability. These types of fluid sources, however, greatly differ in excitation strength from impingement since they are dominated by large separation and gross vortex shedding rather than shear layer instability.

The principle of lock-in with cavities has been more extensively studied with acoustic resonators than structural resonators. In this study, comparisons with the governing physics of the cavity fluid-resonant lock-in case will be made. By measuring the spectral features of the resonator, source excitation and feedback can be tracked. The modal interaction between the cavity source and external resonator are similar to dipole excitation for acoustic resonances. The modal interaction for elastic resonances is not known and will be a major point of study for this investigation. A dipole indicates a force type excitation, but vorticity is a significant factor and the response of the elastic resonance may indicate a moment type excitation. The hydrodynamic force is defined by the crossproduct of the velocity with the vorticity, so the orientation of the dipole over the cavity may not be clear when interacting with an acoustic resonance external to the cavity.

Fundamental understanding of the feedback mechanisms between the shear flow fluctuation over the cavity and structural resonance of an adjoining or nearby structure would extend understanding of the effect of elastic properties on the more commonly encountered fluid-resonant lock-in of cavities. An experimental configuration has been evaluated which provides a platform for developing analytical models for predicting and describing lock-in conditions and the roles of the fluid and structural dynamics.

Significant challenges remain for improving this platform. Interaction of a cavity source with an internal resonator, such as a local wall vibration or a quarter-wave acoustic resonance, may produce stronger coupling than an interaction with an external resonator such as a vibrating elastic beam containing a cavity source. The external resonator is a more distributed mass when coupled with the cavity source compared to an internal resonator which is more discrete when coupled with the cavity source. Fluid-resonant cavity lock-in with a longitudinal pipe acting as a distributed acoustic resonator has been achieved (Rockwell et al, 2003). This type of coupling, however, occurs without fluid density differences between the source and resonator. This investigation is currently determining if fluid-elastic cavity lock-in for a distributed resonator is possible given the challenges associated with the influence of the cavity source strength and the resonator characteristics in any coupling achieved.

The experimental arrangement section will discuss the principles of cavity hydrodynamics, fluid-resonant lock-in, and elastic feedback used to design this test. The experimental results section will discuss the measurements taken thus far and the challenges associated with achieving coupling and lock-in. Parallels to other types of lock-in will be drawn to help

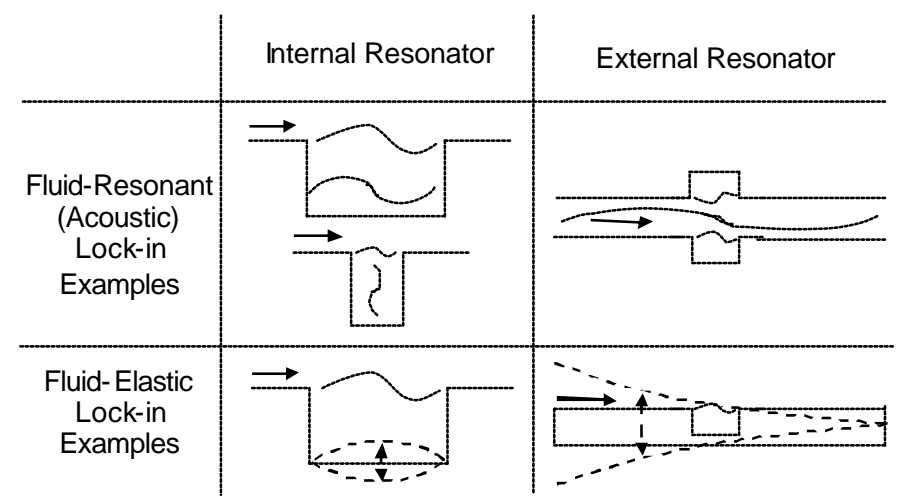

Figure 1: Types of Cavity Lock-in Based on Rockwell and Naudascher (1978) with Resonator Type Distinctions

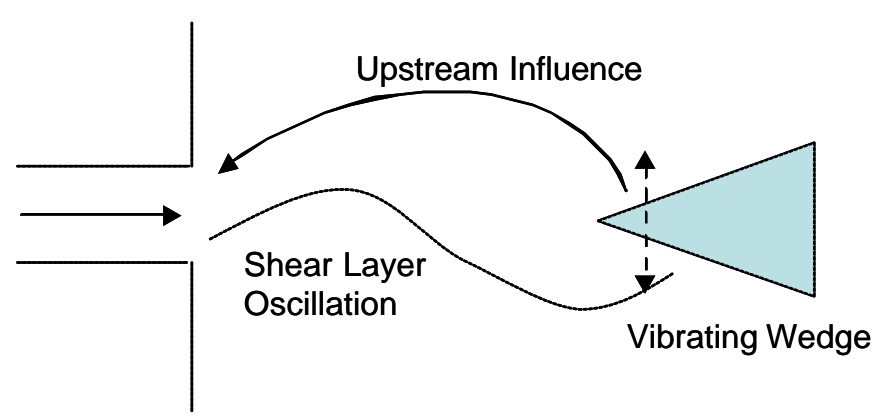

Figure 2: Schematic of Jet-Edge with Vibrating Wedge 
distinguish the controlling mechanism for the type of coupling being studied here. The last section will discuss the steps being taken to determine the influence and importance of the cavity source and elastic resonator characteristics to this very challenging type of lock-in, and to determine the conditions which make lock-in possible.

\section{NOMENCLATURE}

a phase correction

f frequency $(\mathrm{Hz})$

L streamwise cavity length

$\mathrm{n} \quad$ stage of instability (an integer)

$\mathrm{U}$ flow velocity

$\mathrm{U}_{\mathrm{c}}$ convection velocity of vorticity

$\beta$ nondimensionaled frequency of shear layer instability

$\theta \quad$ momentum thickness of shear layer

\section{EXPERIMENTAL ARRANGEMENT}

The design and characterization of an experimental system has been completed to provide a platform for developing an understanding of the feedback mechanism, analytical models for predicting and describing fluid-elastic lock-in conditions, and the roles of hydrodynamics and structural acoustics in the process. The test was designed to accomplish the following objectives:

- Isolation of the predominant instability source to the shear layer fluctuation over the cavity.

- Design for characterization of modal excitation from the shear layer fluctuation.

- Variation of cavity size to identify critical parameters that govern fluid-elastic lock-in.

- Alteration of the inflow boundary layer momentum thickness.

An investigation that explores experimental data and analytical models may reveal the behavior of dynamic structural and fluid characteristics, how they differ from other situations besides cavities, and provide insight into fluidresonant and fluid-dynamic cavity lock-in. The few fluidelastic cavity examples from the 1960's used the cavity walls as the interacting structure with the shear layer over the cavity (Dunham, 1962; Harrington and Dunham, 1960). The natural frequency associated with the walls, however, is dominated by the weight of the water on the structure. This type of resonance is also more discrete when coupling to the cavity source, rather than more distributed as it is for a cavity coupling to a larger structure that contains the cavity. To investigate fluid-elastic

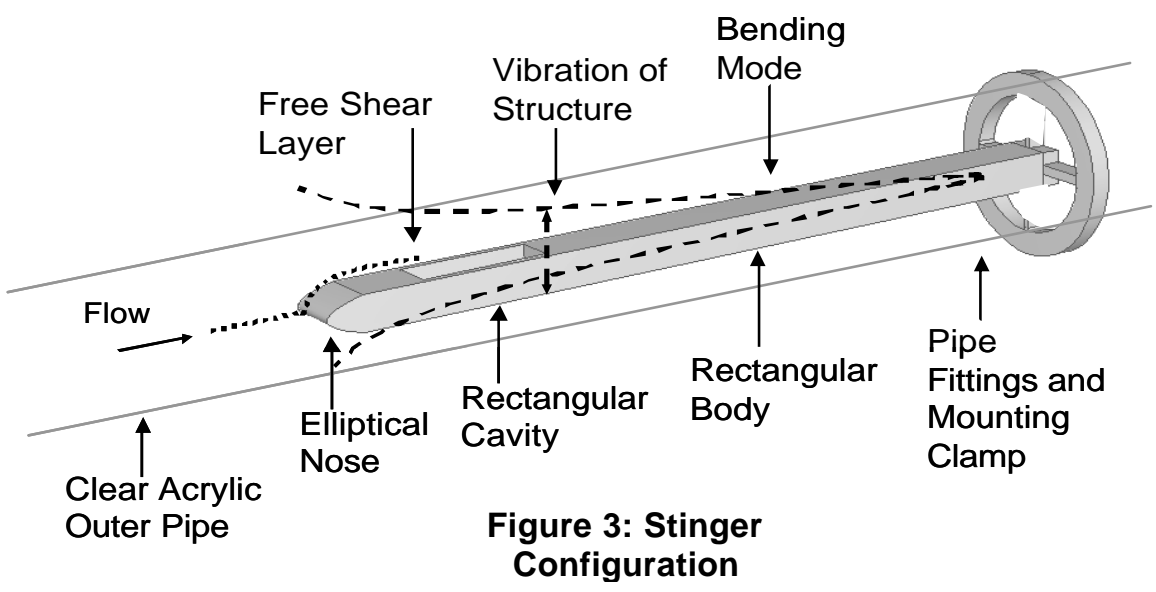

coupling, natural frequencies would ideally be dominated by the structure alone (along with the displaced water mass or added mass). This can be seen in the elastic wedge or blade systems where the elasticity or stiffness of the structure interacts with the impinging flow. Evaluating the response from coupling with a distributed resonator will provide the type of forcing function the cavity source produces.

The configuration in this investigation accomplishes a distributed resonator with a cavity source by using a cantilevered stinger in bending with a milled cavity section (Figure 3). The clamped end of the stinger is captured into the piping using a flange with an integral mounting plate. The stinger cross-section is rectangular to separate the vertical and horizontal bending modes. The cavity shear layer oscillation is excited from flow conveyed over it and should couple to the stinger bending modes.

The stinger with the cavity free shear layer source embedded on a cantilever beam provides bending modes as the preferred elastic resonances for lock-in. This configuration of source and resonator is designed for ease of testing. Interaction with the low frequency bending modes of the system will occur at lower velocities than the other modes. In addition, coupling between this kind of source and the bending modes will show if the coupling is more dominated by the shear layer vertical velocity fluctuation interacting with the first bending mode (antinode), by the shear layer vorticity interacting with the second bending mode (node), or some combination of these like the multiple components of the hydrodynamic force. This overall configuration enables isolation of the source and resonator to the preferred instability and can be adjusted to avoid other instabilities.

The following sections describe how the experiment is designed for instability isolation, cavity source characteristics, elastic resonator characteristics, and other facility system.

\section{Instability Description}

The stinger configuration enables isolation of the source to the cavity shear layer instability and the resonator to the bending modes of the elastic resonances. Avoiding other instabilities and resonators (referred to as interference) is crucial to testing lock-in between the cavity and the elastic resonance. The strength of the interaction is determined by the amount of modal coupling and the other resonances and/or sources present. Lock-in is measured by the amount of energy created in the interaction that is beyond that of the broadband excitation. When lock-in occurs, the mechanism of the source and resonator becomes self-sustaining. When the vortex formation over the cavity length produces separation or a stalled zone, a time delay or phase shift occurs between this area on the stinger and the other areas. The vorticity generated in the separation is considered a time dependent forcing function on the equation of motion. This forcing function is also dependent on the structural motion which results in a net negative damping at the onset of a self-excited oscillation. The only separation or stalled region on the stinger resulting in a significant vorticity should be the cavity.

The stinger is a long slender structure which is cantilevered from a tail that provides a clamped condition. Other possible sources besides the 
cavity flow can occur at the nose, along the body, and at the tail. The nose can be involved in a leading edge instability if the flow separates before the cavity. This is avoided through the use of a 5:1 elliptical nose which is ideal fr a floating stagnation point (Davis, 1999). Several noses with different roughness have been made to vary the amount of inflow momentum thickness to the cavity instability. Trailing edge instability associated with the tail is avoided through a tapering of the geometry.

Resonances in the vicinity of the one of interest would also interfere in the modal coupling. These interferences may come from the support structure and loop itself. Prediction of the facility structural resonances is not needed since the stinger can be adjusted to avoid these interferences. Thus, the bending mode of the stinger is adjustable by adding mass, changing the length of the stinger, or mounting from leaf springs. Acoustic resonances associated with the cavity volume and test section duct have been predicted and are well separated from the stinger resonances. They also have been checked with other possible interference sources in the loop such as the trailing edge flow associated with the tail.

\section{Cavity Source}

The physics of the cavity source should be present regardless of the presence or type of resonator, so the governing physics and parameters associated with fluid-resonant lock-in testing are used in this test design. A full background of the development of these governing physics and parameters are given in Rockwell et al, 2003. A brief description is given here to justify the design of the test.

When the source is a cavity flow, a hydrodynamic instability occurs which is associated with the free shear layer and vorticity across the cavity (Figure 4). At separation, a free shear layer develops and grows which produces oscillatory components. This growth is initially linear and then becomes nonlinear resulting in a transfer of energy into higher frequencies. This shear layer development can be defined both spatially and in time. Small cells of vorticity are associated with the shear layer. As the shear layer grows, the small cells of vorticity cluster together to form a large vortex. Once the shear layer impinges the trailing edge of the cavity, an upstream influence occurs at the shear layer formation point. This hydrodynamic influence is nearly instantaneous for low Mach number flows, and results in a strengthening of the shear layer instability source. It also results in a selection of preferred frequencies for the source, designated as Strouhal stage frequencies. Within the cavity, a backwards flow of circulation is formed from the trailing edge to the leading edge. This circulation has an effect on the upstream influence and is aided by entrainment. The parameter used to describe the shear layer thickness here is momentum thickness. This hydrodynamic instability is categorized as a free shear layer instability and exists without the presence of an external resonator (Lucas et al, 1997).

When an acoustic resonator is present at a frequency which is close to or coincident with the Strouhal source frequency and the phase is aligned, lock-in or synchronization can occur. Figure 5 shows the frequency dependence for increasing velocity of the resonance frequencies (green lines) and the cavity source frequencies (blue lines). This is a compressible

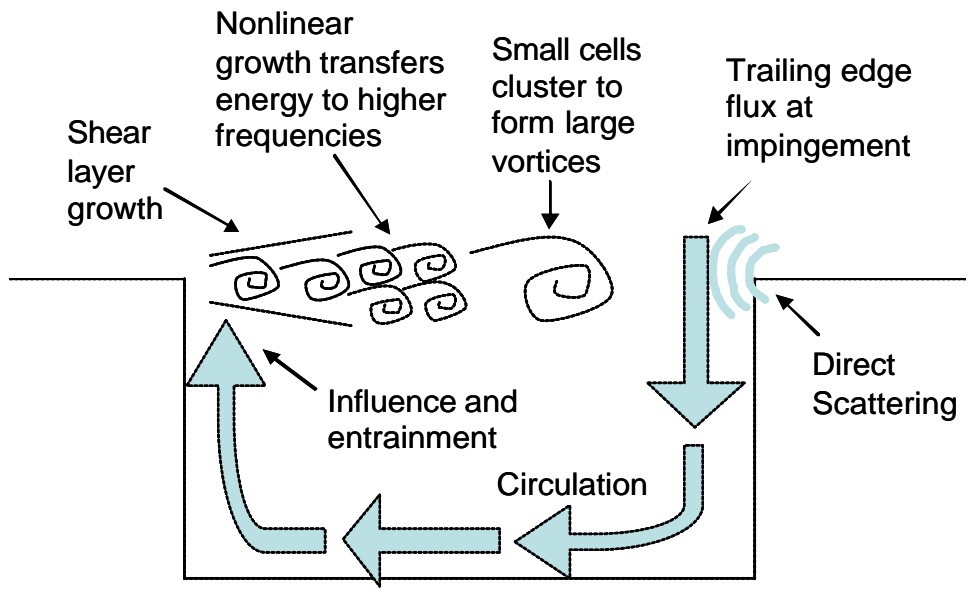

Figure 4: Cavity Oscillation Description (Lucas et al, 1997)

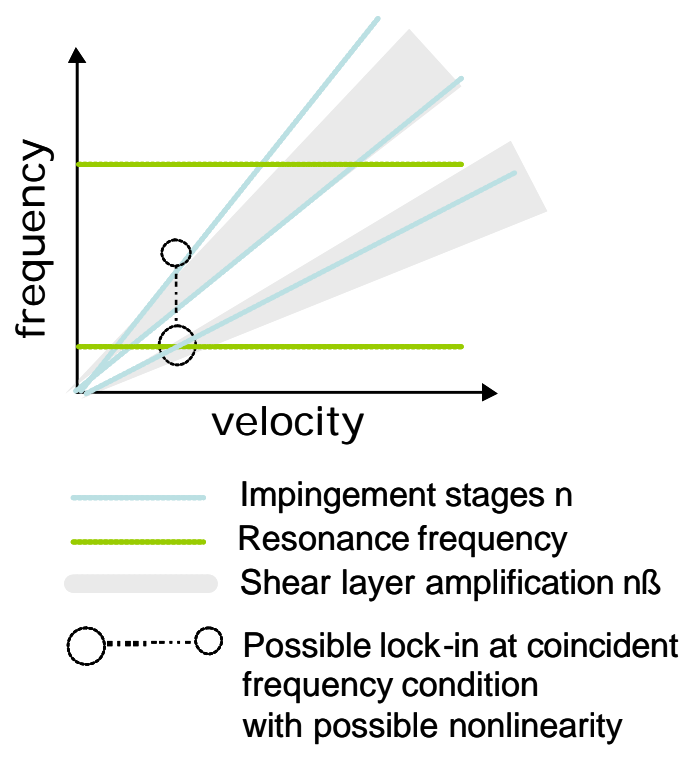

Figure 5: Lock-in Frequencies

feedback process which amplifies the hydrodynamic instability or incompressible feedback process.

The feedforward part of the interaction (excitation of the resonator by the source) is equivalent to a forced vibration of the acoustic resonator by the hydrodynamic fluctuation of the shear layer. The feedback part of the interaction is due to the acoustic fluctuation perturbing the shear layer oscillation. This fluctuation reinforces the shear layer oscillation such that a sustained oscillation may occur. The inclusion of feedback in this process is referred to as lock-in and may result in a flow noise tone. Because of the amount of energy in the resonator and surrounding systems, flow tones often penetrate beyond the resonator and are transferred through fluidborne, airborne and structureborne means.

Considering the cavity source alone, hydrodynamic frequency selection is based on two principles for cavity incompressible feedback. From linearized stability theory, the instability due to the free shear layer may be amplified over a range of $\beta=\mathrm{f} \theta / \mathrm{U}$ from $\sim 0-0.040$ (Figure 5 shaded area). In a cavity, impingement produces a feedback effect based on an 
instantaneous response of the upstream shear layer due to the pressure produced at the impingement. One form predicts nondimensional source frequencies based on the cavity length and the ratio of vortices' convective to freestream velocity: $\mathrm{fL} / \mathrm{U}=(\mathrm{Uc} / \mathrm{U})(\mathrm{n}+/-\mathrm{a})$ (Figure 5 blue lines). There are numerous unresolved issues with this correlation. These parameters are empirically based and can vary depending on the experiment.

Amplification frequencies from the combined contribution of the free shear layer and the impingement are unknown. When the vorticity created in the free shear layer clusters into a large vortical structure, the nonlinear growth of the shear layer produces super-harmonics $(n \beta)$ and sub-harmonics $(\beta / n)$ of the amplification nondimensional frequency. This nonlinear contribution is confirmed in low flow water cavity hydrodynamic experiments (Knisely and Rockwell, 1982; Ziada and Rockwell, 1982).

An additional nonlinear contribution may also contaminate the amplification frequencies. When an external resonator is present, strong interaction between the hydrodynamic oscillation frequencies and the external resonator frequencies may occur, producing additional nonlinearity (Figure 5 extended circle from original intersection point). This results in a strengthening of the cavity source. Strong interaction depends on the modal coupling between the external resonator and hydrodynamic oscillation. Multiple interactions may occur since there are several source stages and resonator modes which can simultaneously interact. Separating these nonlinear effects may not always be feasible with data alone. Visual confirmation of the $\mathrm{n}^{\text {th }}$ stage may indicate where nonlinearity due to $n ß$ and strong lock-in occur alone. Adequate separation of interactions with velocity may indicate a separation of $n ß$ and strong lock-in effects.

The span width of the cavity in this investigation is limited by the width of the stinger. In order to investigate the governing parameters of the lock-in, the cavity depth and length are modified with inserts. The cavity length is adjustable from a maximum length of 2.25 " and a depth of 0.5 ".

A source characterization is completed using laser Doppler velocimetry (LDV). This type of measurement provides a visualization of the flow over the cavity as well as a dynamic single point measurement of the fluctuating velocity. Turbulence statistics such as root-mean-square velocity, intensity, and Reynolds stress can then be generated and plotted against those of other cavity sources and cavity fluid-resonant lock-in investigations for comparison.

\section{Elastic Resonator}

The stinger configuration is modeled as a cantilevered beam with clamped-free boundary conditions. The free end is the nose end which is upstream in the flow. The tail end is the clamped end which is downstream in the flow. Flanges in the duct clamp the tail end. Mass, damping, and the structural modes dominate the characteris tics of this as an oscillator.

Damping cannot be well predicted but is a function of the material and attachments in the structure. More importantly, to characterize the lock-in experimentally, damping must be repeatable. Bolted connections may contribute a significant amount of damping and are minimized for this study. Attachments in the structure occur at the nose/body and body/tail. Welded joints have been considered at these locations, but this would greatly minimize the flexibility of the test configuration since different bodies are used to adjust for cavity placement and overall stinger length. Damping due to the nose/body attachment is not expected since this is an area of low bending stress. Damping due to the body/tail attachment is a significant issue and is evaluated through diagnostic testing. Damping due to vibration in water is also a significant issue and is evaluated in the next section.

The modes of oscillation for a clamped-free beam are very unique (Figure 6). The overtones of the fundamental mode are not harmonics of the fundamental. When driving to generate a pure tone, the overtones will not be present in the result. This is crucial to the investigation of modal coupling. By being able to separate the response due to different modes, a direct modal interaction can be investigated. In other words, when a cavity source stage and elastic bending mode are driving each other, the energy transfer between the source and resonator will be due to that mode alone. No energy can be lost from the source interacting with an overtone of the resonance.

One disadvantage of a clamped-free beam configuration is that the second mode is about six times higher in resonance frequency than the first mode. In order to investigate multiple modes with one source stage, a large velocity range may be needed. This is resolved by testing flexible configurations of varying length cavities and structural modifications and source stages. Figure 7 shows an example of the expected lock-in states for the longes t cavity of 2.25 ".

The cavity is positioned in the stinger to excite the bending modes of the stinger at frequency coincidence. The cavity is nearest the free end of the stinger such that the fluctuating transverse velocity dominates coupling and the first bending

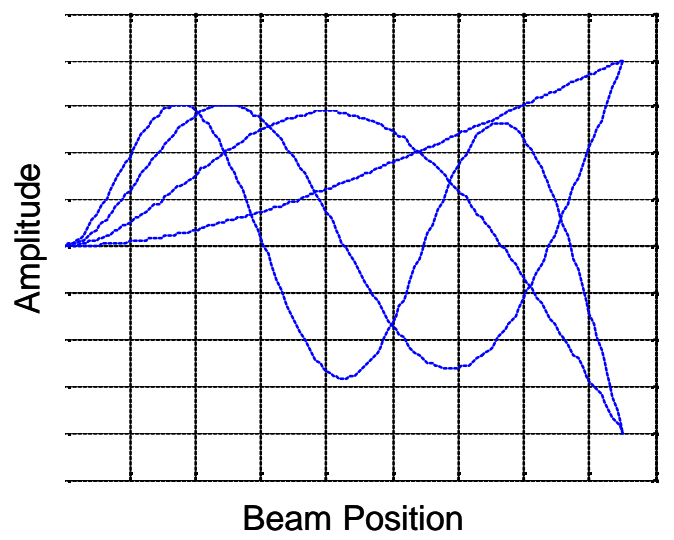

Figure 6: Modes Shapes for Clamped-Free Beam

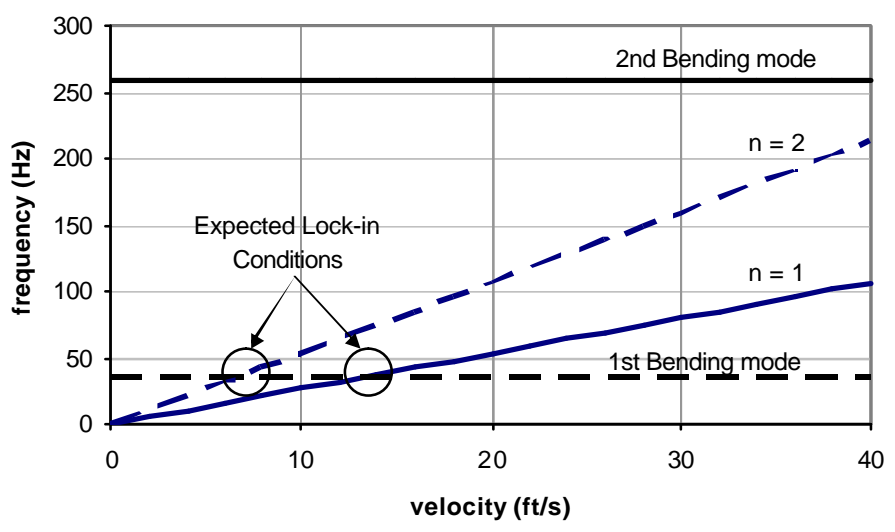

Figure 7: Expected Lock-in Conditions for 2.25" Long Cavity with Bending Modes of Stinger 
mode response will be high at coincident frequency points. If the fluctuating vorticity from the cavity dominates the coupling, the second bending mode response will be high due to the nodal position of the resonator modal displacement where a moment will dominate vibration.

The stinger is embedded with light accelerometers that are potted in four locations along the length in order to measure vibration response. This will add some damping to the system. Phase correlations between the LDV flow data and the accelerometers will be examined to identify coupling as a function of frequency while including source visualization.

\section{Facility Systems}

The test facility is a typical water tunnel. Hoses upstream and downstream of the test section are used to isolate from resonances and noise in the facility from the pump and feed piping. The main duct of the test section is 10' long circular 3" ID cast acrylic duct. The downstream flange is where the stinger is mounted and accessed. A shorter 2' acrylic end is used downstream of this prior to the exit hose to avoid unsteady end effects on the stinger. The test section is mounted to vibration isolation tables to isolate from facility noise. The facility acoustics are monitored in various locations with accelerometers and acoustic pressure transducers. Honeycomb sections and screens are added at various times in the experiment to change the inflow turbulence. A waterbox around the stinger is used at times for LDV measurements.

\section{EXPERIMENT RESULTS}

The vibratory response of the stinger is measured for incrementally changing flow velocity. For example, Figure 8 shows the tip acceleration for increasing flow velocity from about 0 to $\mathfrak{3} \mathrm{ft} / \mathrm{s}$. Although very clean, repeatable linear vibration is achieved, little interaction between the cavity and stinger is measured at the flow states corresponding to frequency coincidence. For example, Figure 9 shows the amplitude of first bending mode response $(\sim 36 \mathrm{~Hz})$ for increasing flow velocity. Variation of amplitude response at a modal frequency with velocity indicates the type of flow induced vibration. The fluid dynamic forcing function can be characterized from the velocity dependence. Nonlinear response with the log of flow is indicative of a lock-in state where the structural response is providing feedback and strengthening with excitation source (Figure 10). Response is fit with the relationship: $\mathrm{dBg}^{2}=\mathrm{C}+10(\mathrm{n}) \log \mathrm{U}$, where $\mathrm{dBg}^{2}$ is the acceleration response, $\mathrm{C}$ is a constant which corresponds to the $\mathrm{y}$-intercept, and $\mathrm{n}$ is a constant associated with the slope of the logarithmic response with velocity $U$ in $\mathrm{ft} / \mathrm{s}$. This is equivalent to $10 \log \mathrm{U}^{\mathrm{n}}$ where $\mathrm{n}$ may indicate the type of excitation. The dashed line curve fit in Figure 9 indicates the small amount of amplification above linear response. For comparison, lines for $\mathrm{n}=3$ and $\mathrm{n}=2.5$ are shown in green and blue, respectively. Figure 11 shows the deviation in response beyond the curve fits. There is very little amplification achieved, and thus little interaction between the stinger and the cavity occurred.

Several different length cavities have been tested such that opportunities for interactions between the first and second stages of the cavity oscillation with the first two bending modes of the stinger have been tested. The small amount of amplification that is measured is small compared to that of a smooth surface (completely filled-in cavity) (Figure 12). The response characteristics appear to be caused by flow sources other than the cavity shear layer instability. A possible instability at the leading edge of the stinger has been ruled out When the stinger is flipped around with respect to the flow velocity condition, the results are comparable to the nominal direction. Interference effects from a small varying angle of attack at the nose are not observed. Effects from a possible disturbance at the nose interfering are not observed.

To explain why lock-in has not been achieved in this configuration, several different considerations related to the source, resonator, and coupling between are discussed in the following sections.

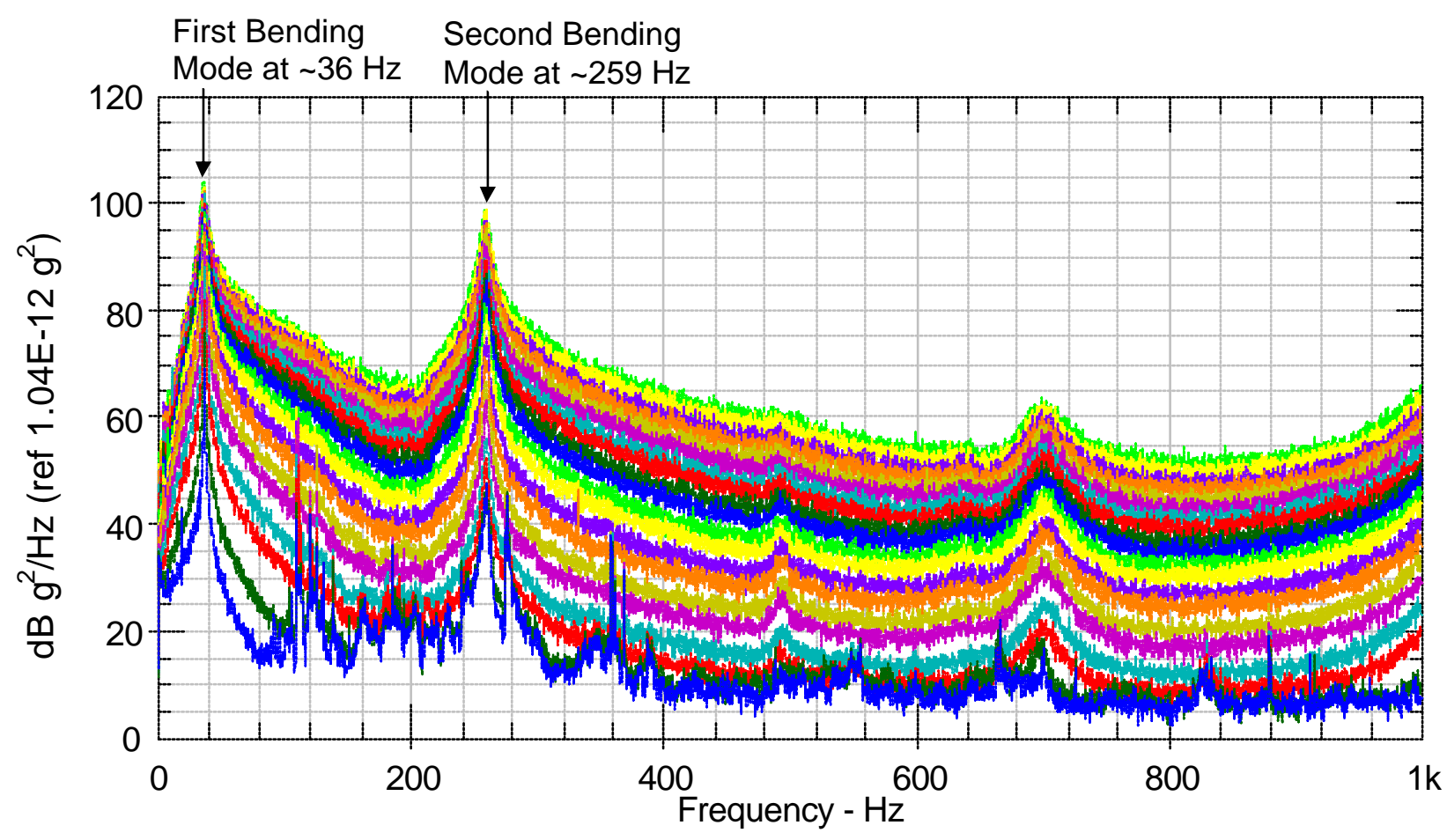

Figure 8: An example of stinger response with 2.25 " cavity at tip with increasing velocity 


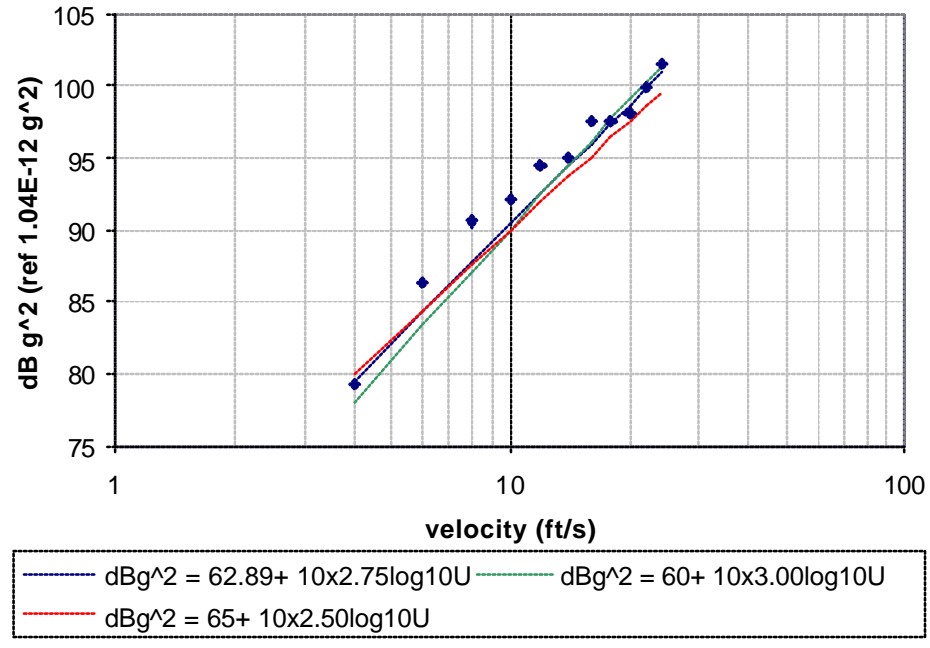

Figure 9: Response of stinger first bending mode measured at tip with various linear fits shown

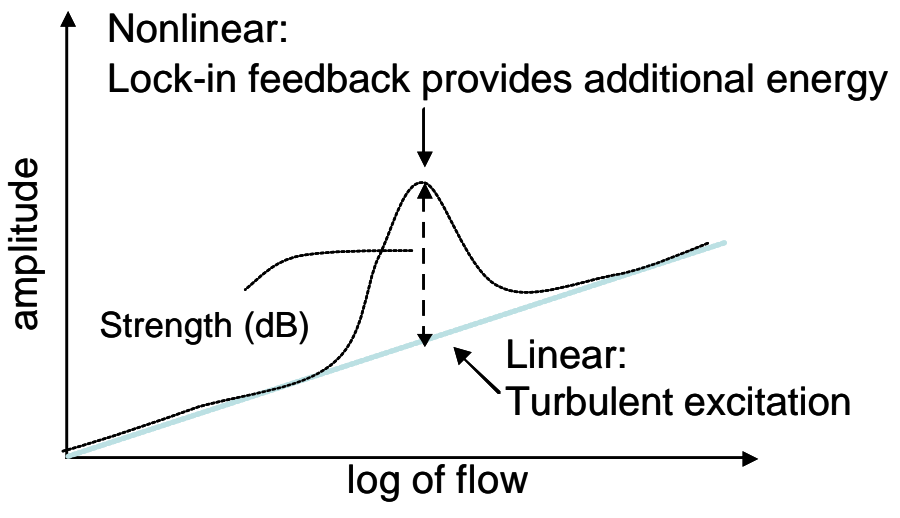

Figure 10: Expected Lock-in Response

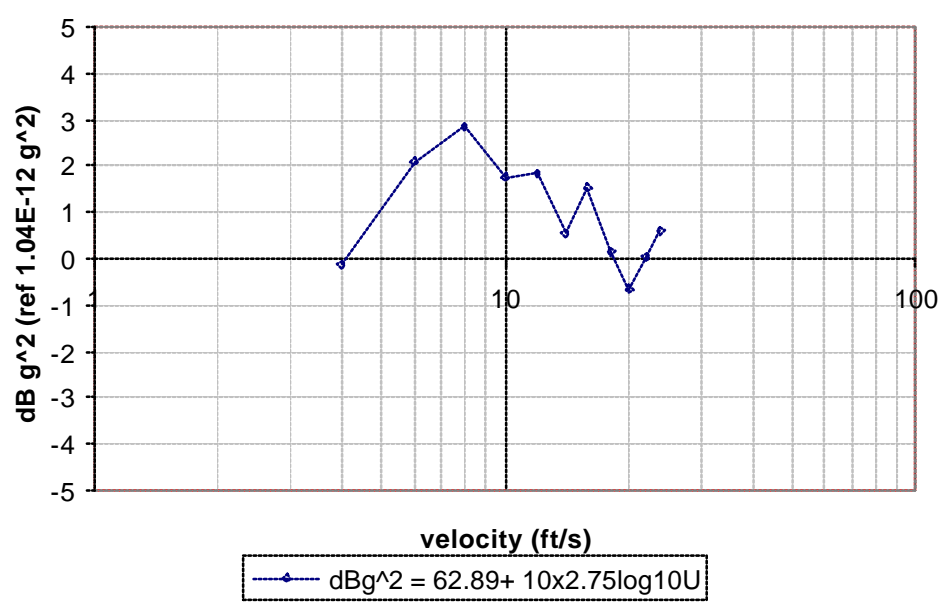

Figure 11: Deviation beyond linear response of stinger first bending mode measured at tip

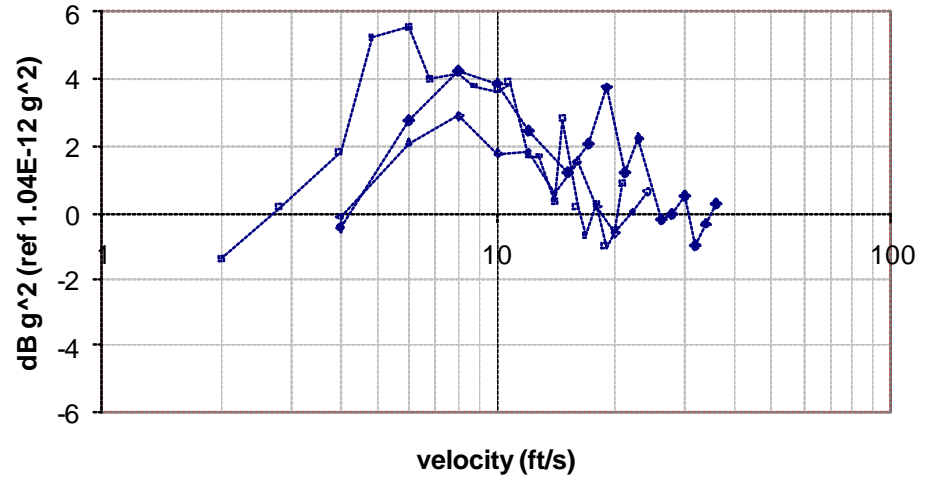

Figure 12: Comparison of stinger amplification with and without cavity (regular and dashed line is with 2.25" long cavity; bold line is smooth surface)
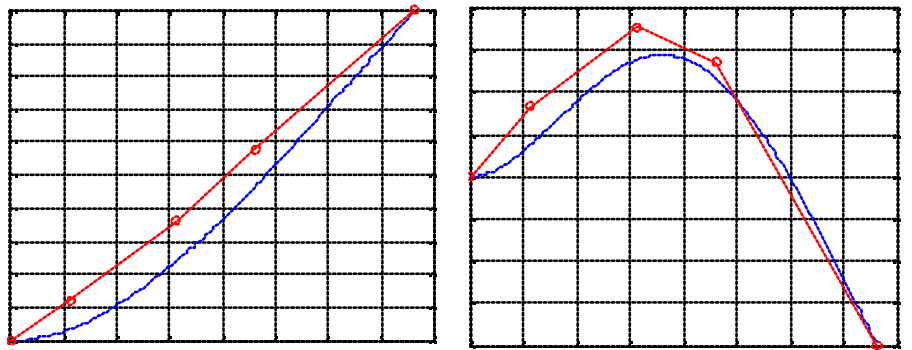

Figure 13: Mode Shapes for the First Bending Mode (Left) and Second Bending Mode (Right); Red line is experimental, blue line is analytical

\section{Insufficient Coupling May $\mathrm{Be}$ Due to High Mass Damping Parameter}

In bluff body lock-in, the mass damping parameter is critical for achieving lock-in. The mass damping parameter is defined as: $\mathrm{m}^{*} \zeta$, where $\mathrm{m}^{*}$ is the ratio of the mass in motion normalized by displaced water mass (added mass) and $\zeta$ is the damping factor. The mass damping parameter in the experiment described here is on the order of $10^{-2}$ which is high compared to bluff body lock-in experiments and is in a region where lock-in may not be achievable. (The limited mass damping parameter will vary for different amounts of mass ratio as well (Khalak and Williamson, 1999).)

The resonance conditions of the stinger were measured from modal testing. The bending modes are typical of those for a cantilevered beam with a significant amount of added mass from the water. This added mass accounts for a larger mass damping parameter than if the resonator had been acoustic and the same flu id density as the cavity source. Wet measurements were obtained from operational mode shapes at low velocity flow. Dry measurements were obtained using modal impact testing. The impact and operational mode shapes are as expected (Figure 13). The first bending mode resonates at about $36 \mathrm{~Hz}$ wet versus $45 \mathrm{~Hz}$ dry. The second bending mode resonates at about $259 \mathrm{~Hz}$ wet versus $355 \mathrm{~Hz}$ dry. The damping for the first mode wet is about $0.9-1 \%$ wet versus $0.5 \%$ dry. The damping for the second mode is about $0.3-0.5 \%$ wet versus $0.3 \%$ dry. This amount of damping is high compared to other elastic vibration experiments and accounts for a larger mass damping parameter. The dry modal mass for the first mode is $0.31 \mathrm{lb}$ compared to $0.3 \mathrm{lb}$ analytically. The dry modal mass for second mode is about $0.33 \mathrm{lb}$ compared to $0.3 \mathrm{lb}$ analytically. The total dry mass of the stinger is about $1 \mathrm{lb}$. 


\section{Insufficient Cavity Source Strength for Stinger Excitation}

LDV measurements show that although a shear layer instability exists at the cavity, it is not strong enough to dominate over broadband turbulence. The pattern of turbulent statistics over the length of the cavity is consistent with many other cavity investigations (Nelson et al. 1981 and 1983; Zoccolla, 2000). The amplitude of the turbulence intensity (TI) is being further studied. In other investigations, TI amplitudes during lock-in are much higher than non-locked in cases.

LDV shows low Reynolds stress which indicates that the vortex generation in the cavity is not coherent enough to generate a strong source. This is limited by the short span of the cavity and the amount of inflow turbulence. The effect of adding Honeycomb filters upstream of the stinger to lower inflow turbulence is being further studied. In bluff body lockin, the source excitation coefficient must be sufficiently high and the mass damping parameter sufficiently low to have an instability. So both the source and the resonator characteristics in the current configuration may be limiting lock-in.

Despite a weak source, inflow conditions for a sufficient shear layer oscillation were achieved. The governing parameter for shear layer oscillations is momentum thickness. The inflow momentum thickness is sufficiently thin, approximately 0.010 " depending on inflow conditions, compared to cavity geometry for strong lock-in conditions from a shear layer instability. This occurs throughout the cavity inflow span. The TI amplitudes also do not vary significantly through the span of the cavity.

\section{IS LOCK-IN OF A CAVITY SOURCE WITH A DISTRIBUTED RESONATOR EVEN POSSIBLE?}

Similar aspect ratio cavities have been tested previously in experiments where lock-in was achieved with the acoustic resonances in the cavity itself and with external acoustic resonances. Coupling with a discrete internal resonator is expected to be stronger than a distributed external resonator due to the limited modal mass from a distributed coupling. Coupling with an acoustic resonator is expected to be stronger than an elastic resonator due to the density difference between water and structure. Both of these effects can be seen in bluff body oscillator models where a criterion of dynamic stability is the net difference between the mass damping parameter and an excitation coefficient related to the coupled source. The mass damping parameter is high in the experiments described here as previously discussed due to the high amount of damping and added mass from vibration in water. Also, the excitation coefficient in the experiments described here is limited due to the limited span of the cavity. So although lock-in is achievable in similar types of resonators with different sources and in similar types of sources with different resonators, lock-in of this combination of cavity source and distributed elastic resonator may not be possible.

The coupling of a cavity source with a distributed resonator is unique from other types of coupling for a few reasons which can be systematically studied. Lock-in with an elastic resonator has been studied with other sources such as bluff bodies and trailing edge instabilities where gross vortex shedding and phasing produce a large amount of excitation. Lock-in with a cavity source has been studied with other resonators such as discrete resonances associated with the cavity and distributed acoustic resonators of the same fluid. The feedforward and feedback parts of the lock-in process can be further studied in this experiment to determine if this type of lock-in is possible along with the influence of the controlling mechanisms. The following sections discuss specific methods to accomplish better coupling by increasing the source strength while decreasing the mass damping parameter.

\section{Increasing Cavity Source Strength}

The lock-in process is composed of a feedforward part which accounts for forced excitation from the cavity onto the structure. Since the cavity is small compared to the wavelength of the stinger vibration, modal interaction is expected to dominate this response. The weak shear layer instability source will be strengthened using a couple of different modifications. Cavities with similar aspect ratios have been studied in fluiddynamic lock-in cases. The cavities are typically mounted in a plate where there are significant side walls bordering the span (cavity is an aperture in the plate). To test this effect, stationary side walls are being added along the sides of the stinger to effectively widen the stinger without changing the existing transverse vibration properties. This will also disrupt any other possible flow instabilities created at the corners of the stinger. In bluff body experiments, span effects have been studied using end plates which disrupt the spanwise coherence of the vortex shedding and the lock-in process. To test this effect, plates will be added to the sides of the stinger that extend above the cavity elevation to effectively channel more flow over the cavity and separate this flow from the side flow along the length of the stinger.

\section{Decreasing Mass Damping Parameter}

The mass damping of the resonator can also be lowered, which should strengthen forced excitation. In bluff body experiments, leaf springs are used to support a cylinder in cross flow in order to produce a low mass damping (Saelim, 1999). To test this effect, a pair of leaf springs will be used to mount the stinger to the tail. Wet measurements of damping will be obtained in stationary flow with a decay method rather than at operational low flow conditions with a bandwidth method. The leaf springs will initially be mounted in an orientation that enables a transverse vibration to the flow, but this can also be done in a streamwise orientation. If lock-in is achieved, changing the orientation of the resonator may provide insight into the orientation of the dipole source with the effect of feedback. The leaf springs can be designed such that either a discrete resonator can be used or a distributed free-free resonator can be used. The leaf springs also provide more isolation from cross-coupling than the tail attachment. Crosscoupling, however, was not seen to be a dominant effect in the experiments so far since the bending modes in the vertical and horizontal orientations are well separated in frequency.

\section{Determining Physics of Feedback Mechanism by Means of External Stimulation}

When the feedforward portion of the lock-in process, or forced excitation, is achieved, it will be possible to further study the feedback portion of the process. Because this is a distributed resonator where the length of the stinger is long compared to the length of the cavity, the feedback of the stinger 
vibration onto the cavity source occurs in a limited volume compared to the entire control volume. Energy from the vibration may be lost to the boundary layer over the entire length of the resonator and may not be sufficient to perturb the cavity shear layer. The effect of the vibrating separation point for the shear layer is also unknown. In controlled cavity lockin experiments, out of phase injection at the shear layer separation is used to attenuate lock-in (Ziada et al, 2002). If the separation point is vibrating sufficiently, a shear layer instability may be generated, but feedback from the vibration may not strengthen it. The location where the vibration perturbs the shear layer may also be important to the feedback process. The impingement end of the cavity may be more important in the feedback process than the separation point (in a jet-wedge it is both). In this experiment, however, the entire cavity is vibrating, which may cause it not to be amenable to feedback effects. To test this effect, amplification of the stinger with a shaker will be done to narrow down the feedback mechanism possibilities. Analytical models such as oscillator models, energy models, and acoustic power generation/dissipation models will be then studied.

\section{CONCLUSIONS}

Lock-in between a cavity source and a cantilevered beam distributed resonator has not yet been achieved. An experimental facility has been designed based on past experiences with cavity lock-in with acoustic resonators and other types of elastic lock-in with different sources such as bluff body lock-in. The current configuration has a spanlimited cavity which does not produce a sufficient source excitation to the stinger vibration. Modifications to strengthen this excitation have been proposed. The mass damping of the current configuration is also high which may preclude lock-in. Modifications to decrease damping have been proposed.

The feedback portion of the lock-in process for a distributed elastic resonator is unique and has not been studied with a cavity shear layer instability as the source. Even with a sufficiently strong cavity source and sufficiently low mass damping resonator, lock-in may still not be possible. Fluiddynamic and fluid-resonant lock-in using span-limited cavities have been studied in the past, but coupling is expected to be stronger for discrete resonators and without density differences for distributed resonators, respectively. There are many aspects of the feedback process that are unknown which make this type of lock-in more challenging if not impossible. Further testing will demonstrate the controlling aspects of this type of lock-in if it is achieved.

\section{REFERENCES}

Davis, M. R., "Design of Flat Plate Leading Edges to Avoid Flow Separation," AIAA Journal (Technical Note), Vol. 18, No. 1, 1979, pp. 598-600.

Dunham, W. H., "Flow-Induced Cavity Resonance in Viscous Compressible and Incompressible Fluids," Report ARC-73, Fourth Symposium on Naval Hydrodynamics, Vol. 3, ONR 1962, 1057-1081.

Harrington, M. C., and Dunham, W. H., "Studies of the Mechanism For Flow-Induced Cavity Resonances," $J$. Acoustical Society of America, Vol. 32, July 1960, p. 921.

Khalak, A. and Williamson, C. H. K., "Motions, Forces, and Mode Transitions in Vortex-Induced Vibrations at Low Mass-Damping," Journal of Fluids and Structures, Vol. 13, 1999, pp. 813-851.

Knisely, C. and Rockwell, D., "Self-Sustaining LowFrequency Components in an Impinging Shear Layer," Journal of Fluid Mechanics, Vol. 116, 1982, pp. 157-186.

Lucas, M. J., Noreen, R. A., Sutherland, L. C., Cole, J. E., and Junger, M. C., Handbook of the Acoustic Characteristics of Turbomachinery Cavities. New York: ASME Press, 1997.

Nelson, P. A., Halliwell, N. A., and Doak, P. E., "Fluid Dynamics of a Flow Excited Resonance, Part I: Experiment," Journal of Sound and Vibration, Vol. 78, 1981, pp. 15-38.

Nelson, P. A., Halliwell, N. A., and Doak, P. E., "Fluid Dynamics of a Flow Excited Resonance, Part II: Flow Acoustic Interaction," Journal of Sound and Vibration, Vol. 91, 1983, pp. 375-402.

Rockwell, D., Lin, J.-C., Oshkai, P., Reiss, M. and Pollack, M., "Shallow Cavity Flow Tone Experiments: Onset of Locked-On States," Journal of Fluids and Structures, Vol. 17, No. 3, March 2003, pp. 381-414.

Rockwell, D. and Naudascher, E., "Review - SelfSustaining Oscillations of Flow past Cavities," ASME Journal of Fluids Engineering, Vol. 100, 1978, pp.152-165.

Saelim, N., Self-Excited Oscillations of a Horizontal Cylinder Adjacent to a Free-Surface, MS Thesis, Lehigh University, 1999.

Ziada, S. and Rockwell, D., "Generation of Higher Harmonics in a Self-Oscillating Mixing Layer-Wedge System," AIAA Journal, Vol. 20, No. 2, 1982, pp. 196-202.

Zoccola, P. J., Experimental Investigation of Flow Induced Cavity Resonance, Ph.D. Thesis, The Catholic University of America, 2000.

Ziada, S., Ng, H., and Blake C., "Flow Excited Resonance of a Confined Shallow Cavity in Low Mach Number Flow and Its Control," ASME, Applied Mechanics Division, Vol. 263, No. 2, 2002, pp. 889-898. 